Development and Implementation of Optimal Filtering in a Virtex FPGA for the Upgrade of the ATLAS LAr

\title{
Calorimeter Readout
}

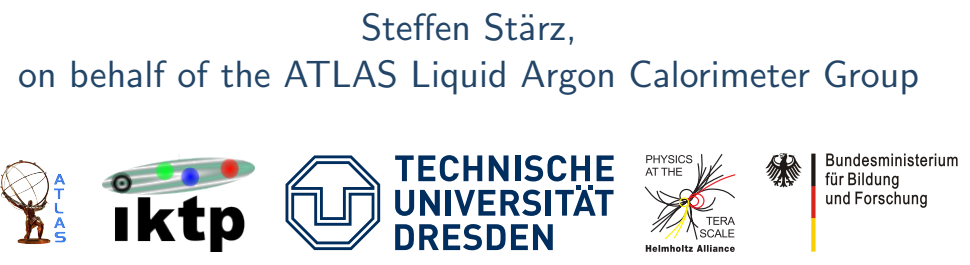

TWEPP 2012, September 18, 2012 


\section{Overview}

(1) Motivation

(2) Signal Filter Requirements

(3) Different Filter solutions

(4) FPGA implementations

(5) Summary and Outlook 


\section{Motivation}

- Upgrade to HL-LHC in two phases $(\approx 2015, \approx 2020)$

- $L=10^{34} \times(1 \rightarrow 2-3 \rightarrow 5-7) \mathrm{cm}^{-2} \mathrm{~s}^{-1}$

$\Rightarrow$ new readout electronics for ATLAS Liquid Argon Calorimeters required

$\Rightarrow$ provide finer granularity to Level-1 trigger system

- 4 individual calorimeter layers, finer segmentation

$\Rightarrow$ improve resolution for trigger objects like electrons,

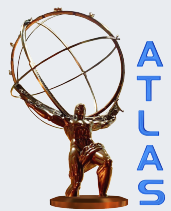
photons, jets, missing transverse and total energy

$\Rightarrow$ reduce trigger rates from background

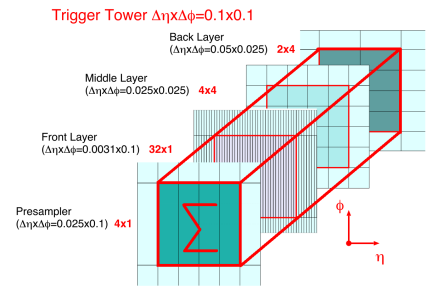

The Challenge

- fully digitized readout (Super Cells)

- $\approx 60000$ trigger readout channels

- $40 \mathrm{MHz}$ sampling rate 


\section{Proposed readout architecture for LAr Barrel and Endcap}

Red: new readout modules for Phase-I; other colours: current readout modules

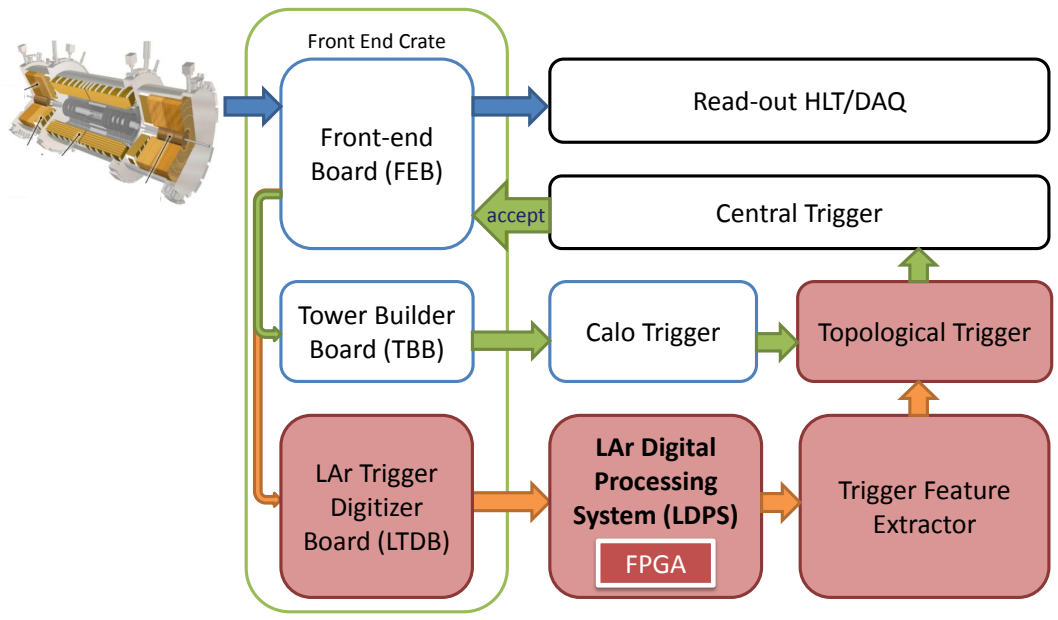




\section{Signal Filter Requirements}
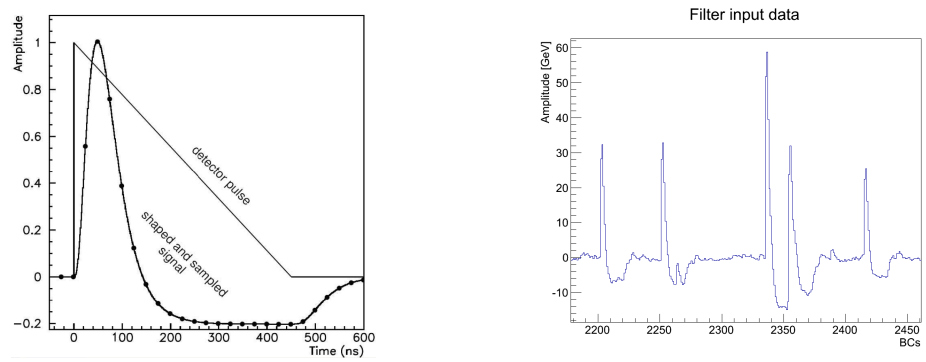

Filter Requirements in Upgrade Scenario

- noise suppression

- correction for out-of-time pile-up

- identification of the correct bunch crossing (BC)

- continuous ADC samples at $40 \mathrm{MHz}$

- tight latency budget ( 6 BCs $\approx 150 \mathrm{~ns}$ ) 


\section{Possible filters}

- adaptive filters: adjust transfer function on the fly

$\Rightarrow$ reference signal required, but relatively long transition time

$\Rightarrow$ massive matrix multiplications

$\rightarrow$ bad for FPGA resources

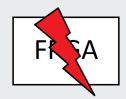

- (non adaptive) FIR filters: calculate coefficients in beforehand

$\Rightarrow$ reference signal required, but stable, fix response delay

$\Rightarrow$ multiply-add structures

$\rightarrow$ good for FPGA resources

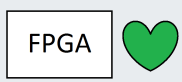

- something in between: IIR filter, Heuristic filter, Wiener filter, ... 


\section{Filter simulation input data generation}

- simulated flat signal energy spectrum: 0 - $80 \mathrm{GeV}$

- pile-up spectrum: 0 - $14 \mathrm{GeV}$ (power-law parametrization [1])

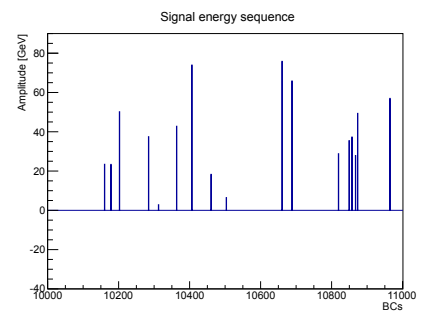

$\times$ pulse shapes from detector simulation
(LAr Super Cells, Barrel, 2nd Layer)

+ noise RMS: $200 \mathrm{MeV}$ [2]

$\Rightarrow$ sequence of $1 \mathrm{M} \mathrm{BCs}$

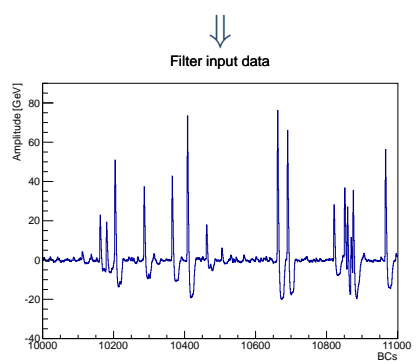

[1] The CMS Collaboration: Measurement of charged hadron spectra in proton-proton collisions at $\sqrt{s}=14 \mathrm{TeV}$

[2] Georges Aad et al: Performance of the electronic readout of the ATLAS liquid argon calorimeters 


\section{Filter simulation results}

simulated input energy spectrum:

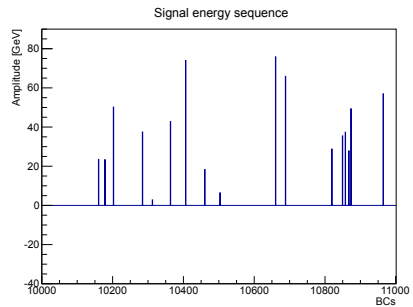

possible filter results:
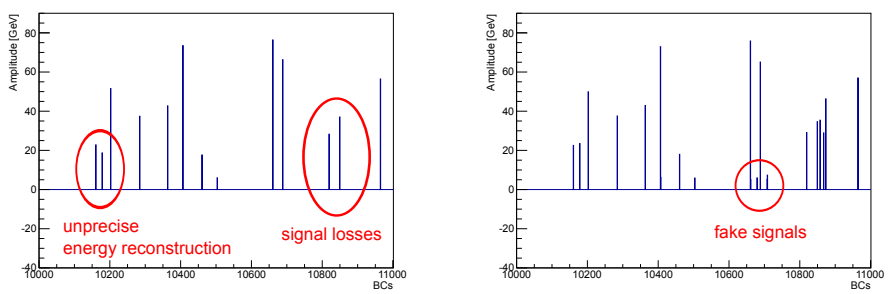


\section{5-staged FIR filter with shape detection}

- similar to current implementation in ATLAS LAr Read-Out Driver

- $E=\sum_{i=1}^{5} a_{i} \cdot\left(S_{i}+\right.$ Ped $\left._{i}\right)$

- shape detection: $E \geq 0$ and at least 4 samples compatible with pulse shape $g_{i}:\left|S_{i}-g_{i} E\right|<\frac{1}{2^{N}}\left|g_{i} E\right|$ (easy to implement in FPGA)
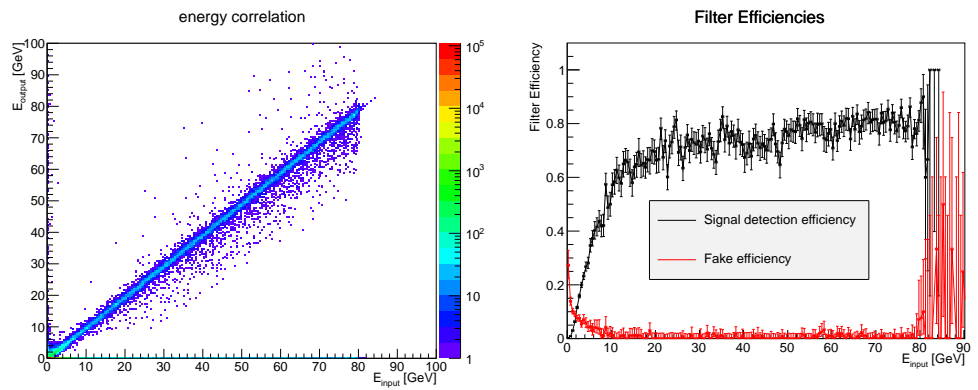

- pile-up enlarges energy spread, poor signal detection efficiency 


\section{FIR filter with shape detection and forward correction}

- if signal found $\rightarrow$ correct subsequent samples for known pulse shape: $S_{i} \rightarrow S_{i}+\sum_{k, j} g_{k, j} E_{k}$
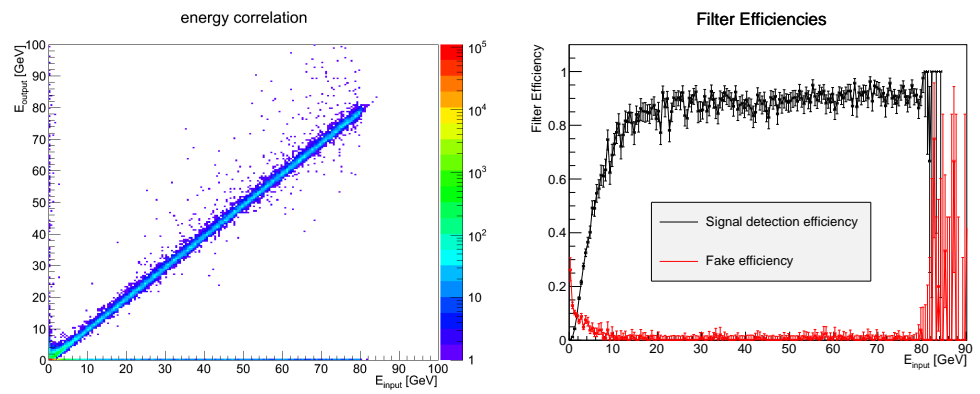

- more precise, improved detection efficiency 


\section{3-stage FIR: peak detection (derivative approach)}

- derivative approach: $E=\frac{w_{1}}{2} \cdot\left(S_{i+1}-S_{i}\right)-\frac{w_{2}}{2} \cdot\left(S_{i}-S_{i-1}\right)$

- weights depend on pulse shape
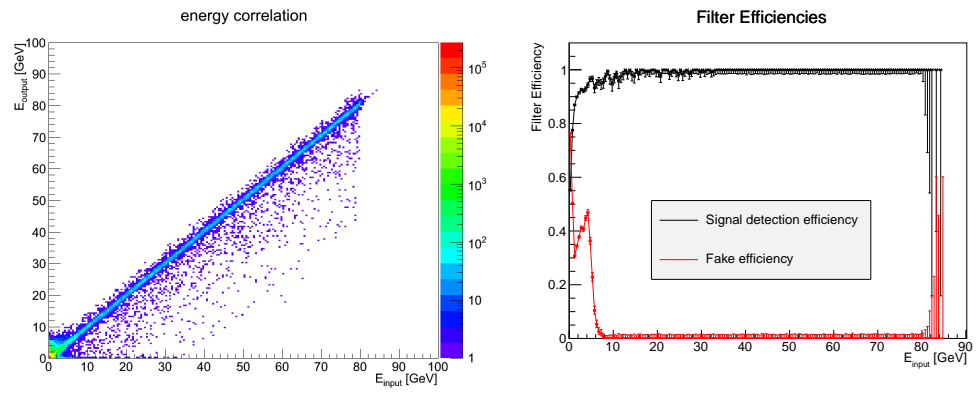

- less precise than FIR with shape detection

- fakes only signals at small amplitudes

- almost no signal losses 


\section{Wiener filter (FIR) with shape correction approach}

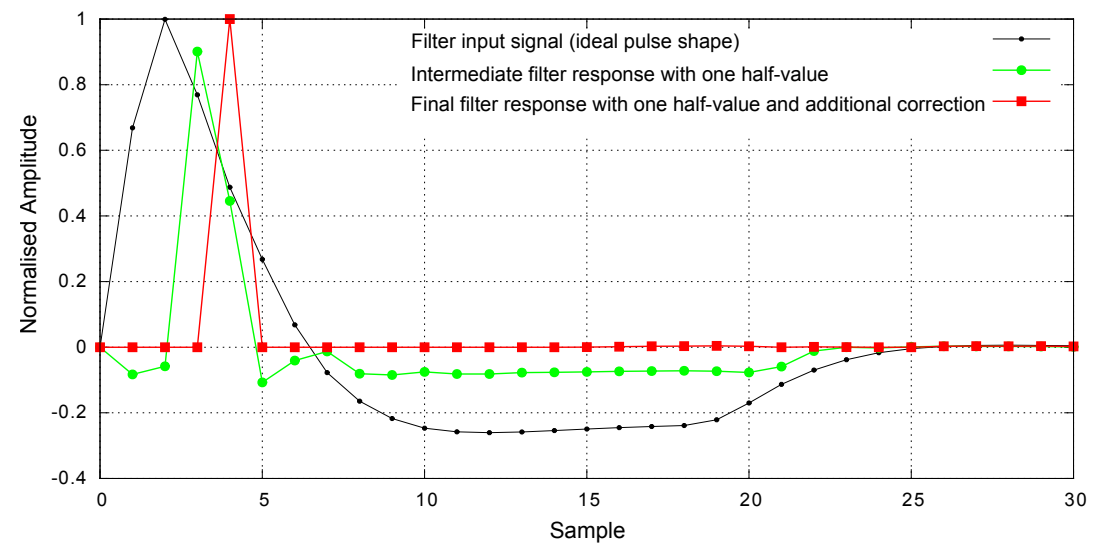

- Final filter response (red) after $\approx 6 \mathrm{BCs}$ (picture shows simulation) 


\section{Wiener filter (FIR) with shape correction approach}

- combines Wiener filter with internal trigger for shape correction (2 small FIRs and shift registers)
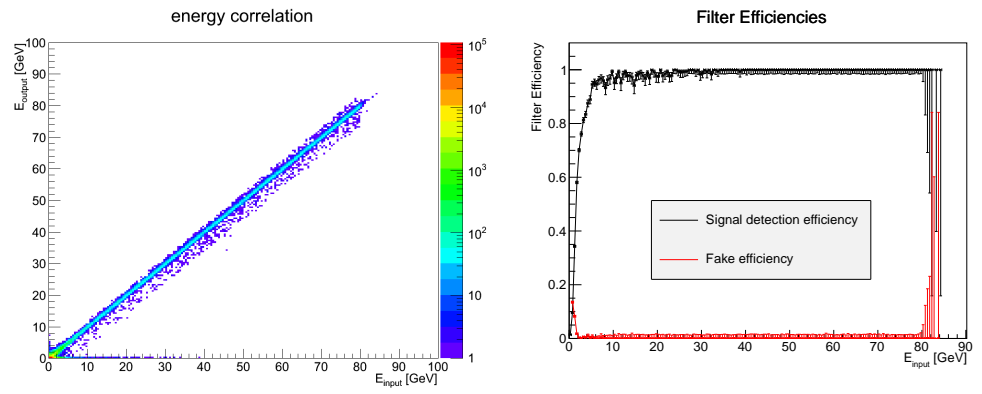

- excellent precision

- no fakes

- almost no losses 


\section{Other filters investigated}

\section{IIR Filter - discarded}

- unstable in filter response (accumulating offset)

\section{Inverse FIR Filter}

- very sensitive to pulse shape $\rightarrow$ second tiny peak (2\%) after 9 BCs

\section{Heuristic Filter}

- pile-up suppression by correcting for the history of up to 8 events in $25 \mathrm{BCs}$ 


\section{Implementation}

- Xilinx Virtex-5, 6 and 7 (ML505, ML605, VC707)
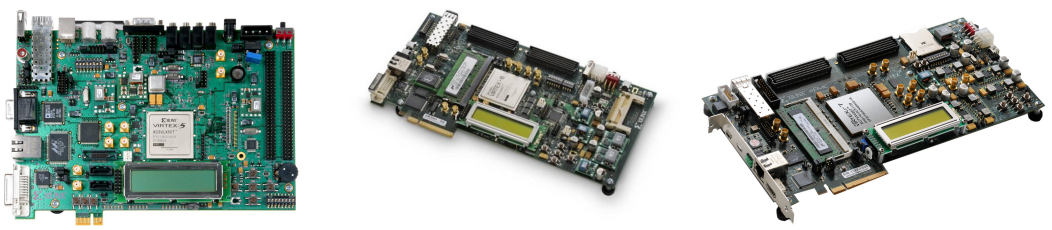

Implementation framework

- Interface with Gigabit Ethernet (UDP/IP)

- Input data buffered in RAM

- Online updatable filter coefficients (SRLC32E) 


\section{Resource utilization}

\section{Resources for 1 filter unit}

\begin{tabular}{c|c|c|c|c} 
filter & DSPs & Slice LUTs & latency (clk/BCs) & max. channels \\
\hline FIR (shape) & 10 & $\approx 400$ & $42 / 5.25$ & $8 * 280$ \\
peak FIR & 3 & ongoing & $28 / 3.5$ & $8 * 933$ \\
Wiener & 16 & $\approx 430$ & $46 / 5.75$ & $8 * 175$
\end{tabular}

- All filters use 8-fold multiplexing (40 MHz LHC vs. $320 \mathrm{MHz}$ FPGA)

- Channel estimates for Xilinx VC707 Evaluation board (VX485T-2 with 2800 DSPs and 75900 Slices)

- ATLAS LAr requirement: 1280 channels per FPGA $\rightarrow$ fulfilled 


\section{Summary and Outlook}

\section{Filter summary}

- Wiener filter with shape correction shows best performance

- FPGA resource utilization varies a lot for different filters, but all implementations fulfill ATLAS LAr requirements

- Latency budget met by all filters by design

\section{Future tasks}

- Simulation with different physics scenarios

- Simulation of different detector regions

- Pulse saturation effects are under study 


\section{The last slide}

Thanks for your attention!

Your feedback is welcome at any time!

Questions? 


\section{Proposed readout architecture for LAr Barrel and Endcap}

Black: current readout modules; Red: new readout modules for Phase-I

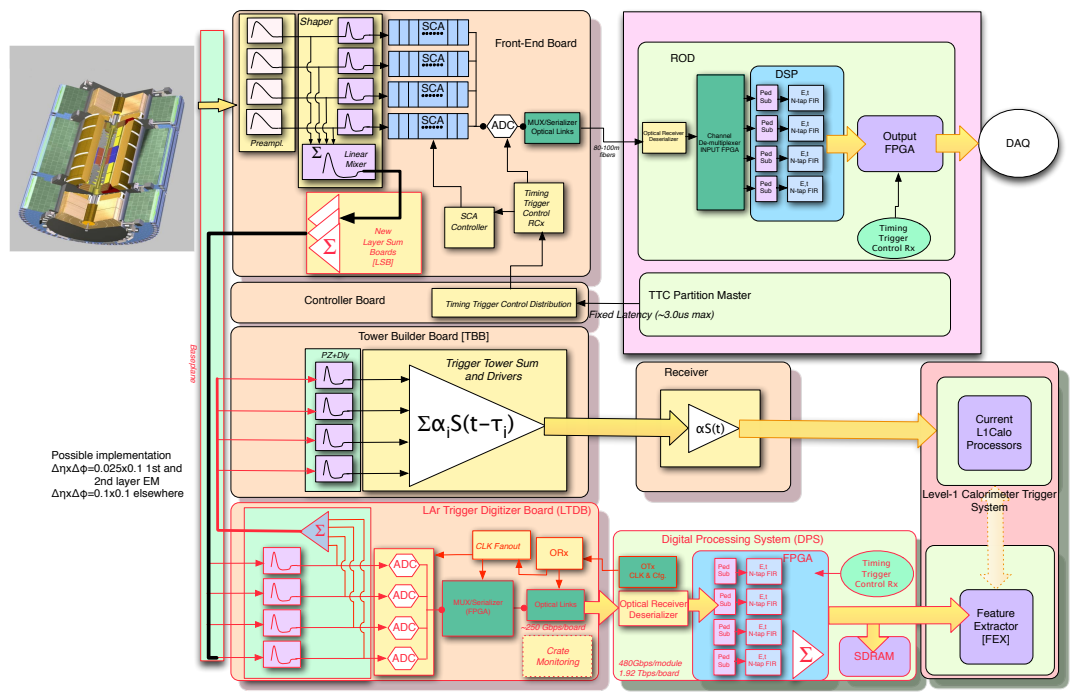




\section{The Wiener filter design in more detail}

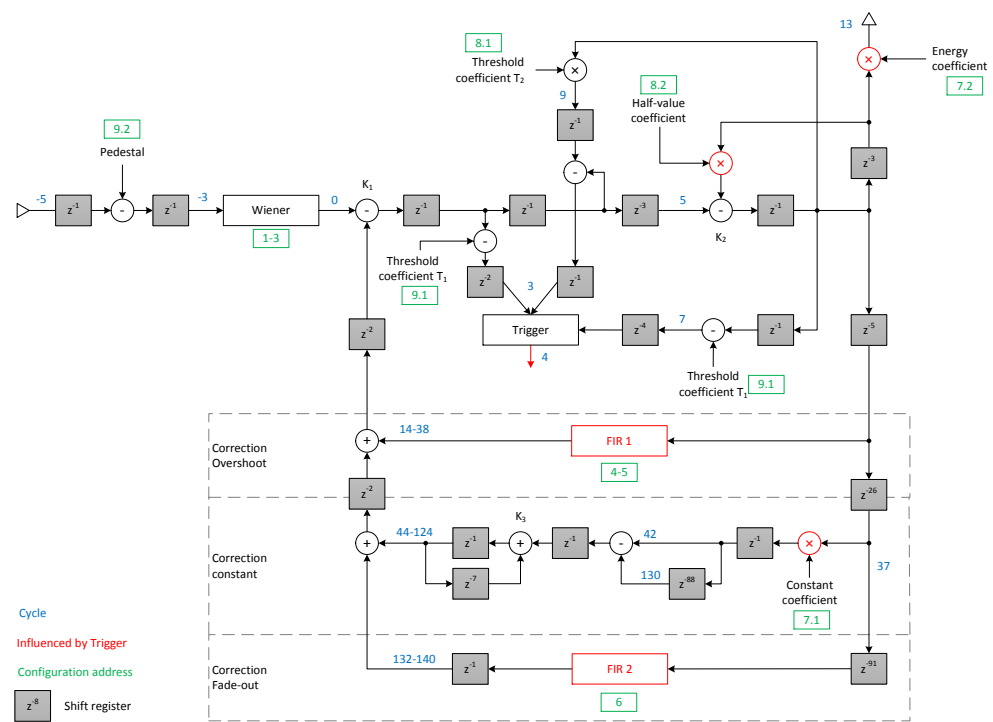




\section{The Wiener filter phase dependence}

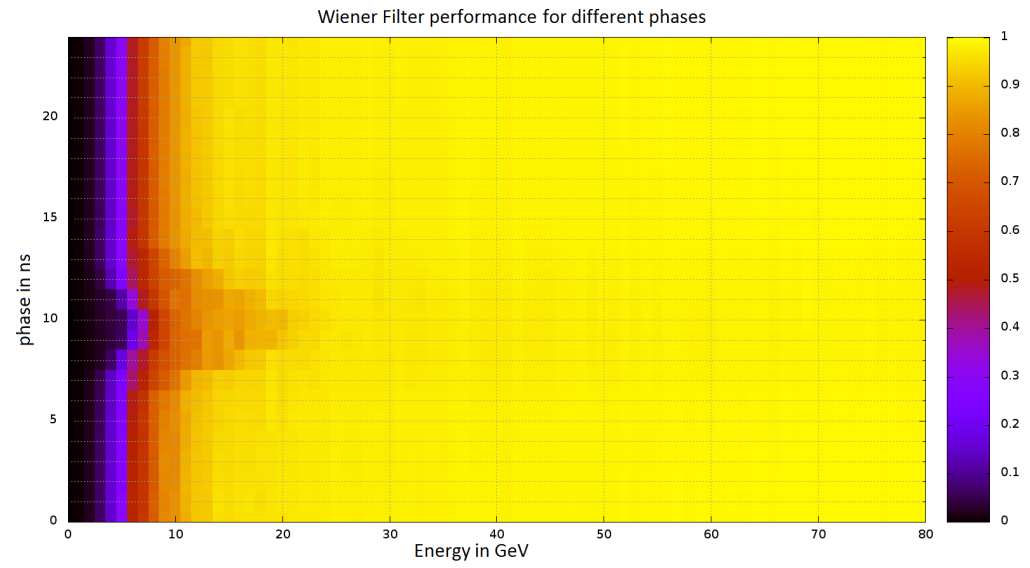

- quality plot: product of precision, inverse fake efficiency and signal detection efficiency 


\section{Coefficients module}

\section{Updatable coefficients}

- requirements:

- instant readout

- rewritable entries

$\rightarrow$ updatable LUT

- solution:

- SRLC32E module

- side-effect:

- 32 addresses minimum

- bitwise data input

- changing bit order

$\rightarrow$ offline calculation

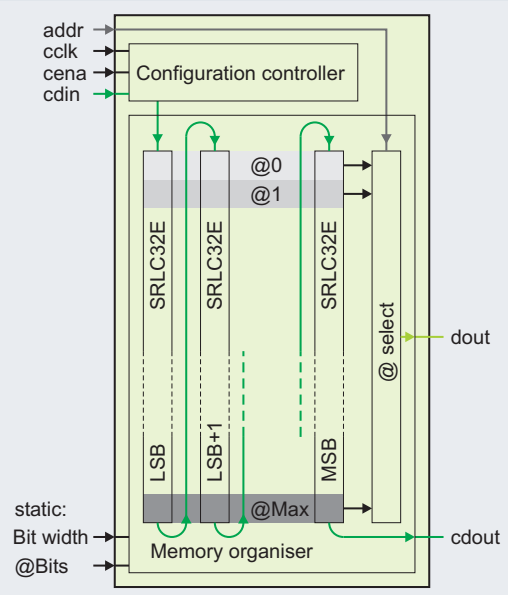




\section{Saturation effects}

\section{Some thoughts}

- Saturation: pulse changes shape $\rightarrow$ filters fail or suffer in performance

- Important for trigger: still identify correct BC

- energy reconstruction becomes less important in saturated region

- currently discussed: combination of linear and non-linear amplification region
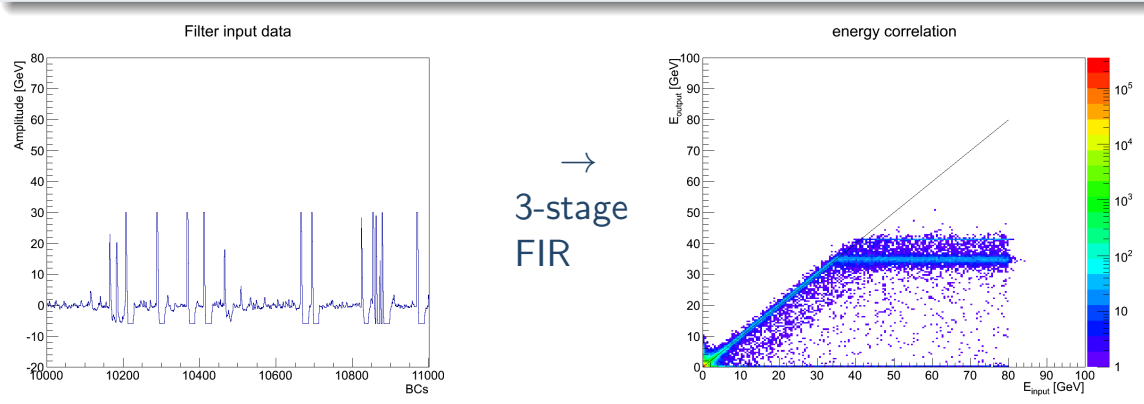


\section{References}

The CMS Collaboration: Measurement of charged hadron spectra in proton-proton collisions at $\sqrt{s}=14 \mathrm{TeV}$

粗 Georges Aad et al.: Performance of the electronic readout of the ATLAS liquid argon calorimeters 